\title{
Effects of an Intraparenchymal Injection of Lidocaine in the Rat Cervical Spinal Cord
}

\author{
María S. Sisti ${ }^{1,2} \cdot$ Carolina N. Zanuzzi $^{1,2,3} \cdot$ Fabián Nishida $^{1,2} \cdot$ Rodolfo J. C. Cantet $^{2,4} \cdot$ Enrique L. Portiansky $^{1,2}$
}

Received: 24 May 2018 / Revised: 30 August 2018 / Accepted: 4 September 2018 / Published online: 8 September 2018

(c) Springer Science+Business Media, LLC, part of Springer Nature 2018

\begin{abstract}
Lidocaine effects in the spinal cord have been extensively investigated over the years. Although the intrathecal route is usually used to treat insults occurring in the spinal cord, the local delivery drug via intraparenchymal infusions has gained increasing favor for the treatment of some neurodegenerative disorders. The aim of the present study was to evaluate the behavioral and tissue effects of the intraparenchymal injection of different concentrations of lidocaine into the rat cervical spinal cord. Young male Sprague-Dawley rats were intraparenchymally injected with $0.5 \%, 1 \%$ or $2 \%$ lidocaine at the C5 segment of the spinal cord. Other rats were injected with saline solution (sham group). Hot plate test was determined at $0,1,2,3,7$ and 14 postinjection (pi) days. Rats of each experimental group were euthanized either at 1,2,3,7 or 14 pi days. Intact animals were used as controls. Sections of the $\mathrm{C} 5$ segment were used for histological, immunohistochemical or immunofluorescence analysis. Injection of $0.5 \%$ lidocaine did not affect neuronal counting, did not evoke an inflammatory reaction, nor induce astrocyte activation. Therefore, a concentration of $0.5 \%$ lidocaine is suggested to promote anti-inflammatory effects after injury.
\end{abstract}

Keywords Spinal cord injury · Local anesthetic $\cdot$ Drug delivery $\cdot$ Toxic effect

\section{Introduction}

Most spinal cord injuries (SCI) are accompanied by additional peripheral tissue damage that frequently alters pain fibers [1] and activates the "pain matrix" at the Central Nervous System (CNS) [2]. Nociceptive stimulation after injury increases the extent of secondary injury, reduces locomotor recovery, increases mortality and tissue loss [1]. Lidocaine has analgesic effect and anti-hyperalgesic

María S. Sisti and Carolina N. Zanuzzi: identical contribution.

María S. Sisti

msusanasisti@gmail.com

1 Image Analysis Laboratory, School of Veterinary Sciences, National University of La Plata (UNLP), Calles 60 y 118, La Plata, 1900 Buenos Aires, Argentina

2 National Research Council of Science and Technology (CONICET), Buenos Aires, Argentina

3 Chair of Histology and Embryology, School of Veterinary Sciences, National University of La Plata, Buenos Aires, Argentina

4 Department of Animal Science, College of Agriculture, University of Buenos Aires, INPA CONICET, Buenos Aires, Argentina and anti-inflammatory properties that greatly contribute to reduce nociception, postoperative pain and analgesic requirements after surgery [3]. Local administration of lidocaine at clinical concentration (2\%) largely inhibits activation of the CNS pain matrix [2].

Over the years, lidocaine effects in the spinal cord have been extensively investigated. Systemic administration of lidocaine after mechanical lesion has shown to improve the neurological outcome of cats with experimental SCI [4] and to reduce the incidence of lumbar SCI in rats [5]. It is also a useful tool for pain management therapies [6,7]. Pre-incisional intrathecal administration of local anesthetics inhibits nerve impulses in response to noxious stimuli and reduces postoperative morphine requirements, while intrathecal lidocaine after peripheral nerve injury reverses tactile allodynia [8].

Although the intrathecal route is usually used to treat insults occurring at the spinal cord [9], local drug delivery via intraparenchymal infusions has gained increased interest for the treatment of some neurodegenerative disorders [10].

Intraparenchymal injection is an invasive procedure but allows direct distribution of therapeutic agents at a specific concentration to target areas or specific cellular populations within them, bypassing the blood brain barrier [10-13]. 
To our knowledge, no tests have been previously done using the intraparenchymal route to deliver lidocaine into the spinal cord. Therefore, the aim of the present work was to evaluate the behavioral and tissular effects of the intraparenchymal injection of different concentrations of lidocaine into the rat cervical spinal cord. All data collected using this approach are expected to serve as a starting point for supporting subsequent therapeutic protocols using the intraparenchymal via for delivering infusions, or as a reference for procedures using intrathecal or other alternative routes.

\section{Methods}

\section{Animals}

Young (3-5-month-old, 250-450 g) male Sprague-Dawley rats $(n=96)$, raised in our rat colony, were used. Animals were housed in a temperature-controlled room $\left(22 \pm 2{ }^{\circ} \mathrm{C}\right)$ on a 12:12 h light/dark cycle. Food and water were available ad libitum. All experiments with animals were performed according to the recommendations of the Guide for the Care and Use of Laboratory Animals of the National Institutes of Health (NIH Publications No. 80-23, revised in 1996). The protocol was approved by the School of Veterinary Sciences, National University of La Plata Institutional Committee for Care and Use of Laboratory Animals (CICUAL), Code 45-114T. All efforts were made to minimize the number of animals used and their suffering.

\section{Handling of Animals and Drug Injection}

Control animals $(n=6)$ were those that allowed us to establish the baseline data for cell counting, determination of white and gray matter areas and to establish similarities between both ipsilateral and contralateral sides of the spinal cord segments. Sham-operated animals $(n=15,3$ rats per time point) were used to establish the effects of the injection needle and of drug vehicle on the spinal cord tissue and cells. The remaining animals were considered as experimental.

Animals were injected either with lidocaine $2.0 \%$ (L2 group), $1.0 \%$ (L1 group) or $0.5 \%$ (L05 group), or saline (sham group). Immediately before surgery, $1.0 \%$ and $0.5 \%$ concentrations of lidocaine were obtained by serially diluting the $2.0 \%$ commercial presentation (Xylocaine ${ }^{\circledR} 2 \%$, AstraZeneca, Haedo, Buenos Aires, Argentina) in 0.9\% saline solution and kept at $4{ }^{\circ} \mathrm{C}$ until use.

On experimental day 0 , rats $(n=75,25$ rats per experimental group and 5 per time point) were anesthetized with ketamine hydrochloride $(40 \mathrm{mg} / \mathrm{kg}$; i.p.) plus xylazine $(8 \mathrm{mg} / \mathrm{kg}$; i.m.) and placed in prone position. All drugs were injected via intraparenchymal route at the $\mathrm{C} 5$ segment of the spinal cord following the protocol described by Nishida et al. [14]. Five microliters either of the lidocaine solution or saline solution were discharged at a rate of $1 \mu \mathrm{l} / \mathrm{min}$. After surgery, animals were returned to their cages and checked periodically until they woke up. As C3, C4 and C5 segments contribute with the formation of the phrenic nerve, attention was paid to respiratory behavior of injected animals.

\section{Heat Sensitivity Test}

The hot-plate test was carried out according to a method previously described [15], at the beginning of the experiment (day 0 ) and at each analyzed time point (days 1, 2, 3, 7 , and 14 pi) until euthanasia. The hot-plate device was set at $55 \pm 1^{\circ} \mathrm{C}$, and animals were placed on a $15 \mathrm{~cm}$ diameter heated surface surrounded by four acrylic walls. The time (measured in sec) between placement (time zero) and licking of their forepaws or jumping (whichever occurred first) was recorded and considered as the response latency. A $20 \mathrm{~s}$ cut-off was used to prevent tissue damage of non-sensitive animals. Three measurements at 2-min-intervals were taken per session.

\section{Euthanasia, Specimen Collection and Processing}

Immediately before euthanasia rats were placed under general anesthesia by injection of ketamine hydrochloride $(40 \mathrm{mg} / \mathrm{kg}$, i.p.) plus xylazine $(8 \mathrm{mg} / \mathrm{kg}$; i.m.) and then intracardially perfused with $300 \mathrm{ml}$ of phosphate buffered saline (PBS) followed by $300 \mathrm{ml}$ of PBS-4\% paraformaldehyde solution.

Animals of lidocaine-injected (L1, L2, L05) and sham groups were euthanized ( 5 or 3 per experimental group, respectively) either at $1,2,3,7$ or 14 days post-injection (pi). Six non-operated rats were used as controls. Three of them were euthanized on day 0 and used for neuronal counting. The remaining control animals were euthanized on day 14 but also were previously used for the clinical assessment at the times indicated for the other groups.

After perfusion, the vertebral column was removed and post-fixed in $10 \%$ buffered formaldehyde for $24 \mathrm{~h}$. The spinal cord was then dissected, immersed in cryopreservation buffer (30\% sucrose; $1 \%$ polyinylpyrrolidone; $30 \%$ ethylene glycol; $1 \% 1 \mathrm{M}$ phosphate buffer; DW to $100 \mathrm{ml}$ ) and stored at $-20{ }^{\circ} \mathrm{C}$ until use. Coronal sections of cervical segments were embedded in $0.5 \mathrm{ml}$ of jellifying solution [10\% sucrose in $1 \mathrm{M}$ phosphate buffer; $4 \%$ low melting point agarose (Sigma Chemical Co., St. Louis, MO, USA)]. After $24 \mathrm{~h}$ storage at $4{ }^{\circ} \mathrm{C}$ the jelly blocks were serially cut into $20 \mu \mathrm{m}$ thick coronal sections using a vibratome (Leica VT 1000S, Germany). Sections were then mounted on jellified slides $(6 \mathrm{~g}$ unflavored gelatin; $0.5 \mathrm{~g} \mathrm{KCr}(\mathrm{SO} 4)_{2} .12 \mathrm{H}_{2} \mathrm{O}$, DW to $300 \mathrm{ml}$ ) and stained with 
cresyl violet for using them for histopathological and morphometric analysis. From each block, three to five sections, $120 \mu \mathrm{m}$ apart, were analyzed.

\section{Immunohistochemistry and Immunofluorescence}

Immunohistochemistry was used for cell counting and to analyze neuronal distribution. $\mathrm{C} 5$ spinal cord sections were mounted on jellified slides and incubated with $0.03 \% \mathrm{H}_{2} \mathrm{O}_{2}$ in PBS for $30 \mathrm{~min}$ at room temperature. Sections were then rinsed twice in PBS containing 0.05\% Tween 20 (PBS-T) and exposed to microwave antigen retrieval using a buffered citrate solution, $\mathrm{pH}$ 6.0. Later, sections were washed twice in PBS-T and incubated with $1 \%$ bovine serum albumin (BSA) in PBS for $30 \mathrm{~min}$, followed by overnight incubation with an anti-NeuN monoclonal antibody (1:200, Millipore, CA, USA) for identification of neurons, or anti-GFAP polyclonal anti-rabbit antibody (Dako Denmark, ready to use) for identification of astrocytes.

The EnVision detection system + HRP (horse radish peroxidase) system labelled anti-mouse or anti-rabbit (DakoCytomation) was applied during $45 \mathrm{~min}$. Liquid 3,3-diaminobenzidine tetra-hydrochloride (DAB) (Vector Laboratories Inc., CA, USA) was used as chromogen and Hill's hematoxylin was used for counterstaining. Control negative sections were prepared by omitting primary antibody.

In addition, immunofluorescence was used to identify neurons. For this purpose, sections were incubated with Neu antibody (1:200). After washing, slides were incubated with 1:1000 Alexa Fluor 488-conjugated anti-mouse secondary antibodies (Invitrogen, Thermo Fisher Scientific Inc.) for $45 \mathrm{~min}$. Counterstaining was done using the fluorescent DNA stain 4',6-diamidino-2-phenylindole (DAPI) during $15 \mathrm{~min}$. FluorSave Reagent (Calbiochem) was used for aqueous mounting. Control negative sections were prepared by omitting the primary antibody. Images were obtained using a scanning laser confocal microscope (Olympus FV1000, Japan).

\section{Image Analysis}

Images of immunohistochemically (two-dimensional analysis) stained C5 spinal cord sections were captured using a digital RGB video camera (Olympus DP73, Japan) attached to a microscope (Olympus BX53, Japan). To create a complete map of the entire C5 segment taken with a $40 \times$ objective, images were captured using a digital image analyzer (cellSens Dimension, V1.7, Olympus Corporation, Japan) and stitched them using an automatic Multiple Image Alignment process. No further processing was necessary after obtaining the original images.

\section{Morphometry}

For morphometric measurements, the Count/Measure option of the image analysis software (ImagePro Plus-v6.3, Media Cybernetics, MA, USA) was used. For determining changes in the affected segment, variations in the ipsilateral and contralateral sides areas on cresyl violet stained sections were considered. For astroglia estimation as an indicator of inflammatory process, GFAP inmmunostained area was considered.

\section{Neuronal Counting}

To determine the total number of neuronal bodies per section, color segmentation on NeuN immunostained sections was performed [16]. Blind quantitative analysis was performed independently by at least two observers, to obtain a more objective counting.

Confocal images (three-dimensional analysis) were used to estimate the number of cells present per segment using the following formula [17]:

$N=\frac{d}{n s} \sum_{i=1}^{n} x_{i}$

where, $N=$ total estimated number of cellular bodies; $d=$ length $(\mu \mathrm{m})$ of the rostro-caudal axis of the cervical segment being assessed $(2 \mathrm{~mm}) ; \mathrm{n}=$ number of non-contiguous $(120 \mu \mathrm{m}$ apart) slices counted per cervical segment $(n=5)$; $s=$ thickness of the section $(20 \mu \mathrm{m}) ; x=$ number of perikarya counted per non-contiguous slice assessed. Therefore, $N$ represents approximately the total number of neurons present in the cervical segment.

\section{Statistical Analysis}

The heat sensitivity test was analyzed as repeated measures on the same animal at different times [18]. The model was fitted with SAS software for mixed models (Proc Mixed and Proc Glimmix, SAS v.9.3; SAS Institute Inc., Cary NC, USA). The response variable time of latency $(T)$ was first checked for normality and the distribution was found to be skewed to the right (higher frequency of larger values). Therefore, the variable $T^{*}=\ln (T)$ was used to build the models estimates and test the hypotheses. The model that best fitted the data was an analysis of heterogeneous covariance with treatments as a classification variable and the covariate time ( $t$, in days) nested within treatments, whereas the error variance was found to be heterogeneous due to treatments.

The remaining data were analyzed by one-way analysis of variance (ANOVA) and Holm Sidak's test for multiple comparisons was used as a post-hoc test. Significance was 
assumed at values of $P<0.05$. All these tests were analyzed using the statistical program GraphPad Prism version 6.00 for Windows (Graph Pad Software, San Diego, California, USA, http://www.graphpad.com).

\section{Results}

None of the injected rats showed abnormal respiratory frequency, produced respiratory noises or adopted orthopneic stand at neither time points analyzed. In no case, animals required manual emptying of the bladder.

\section{Heat Sensitivity Test}

No significant differences were detected between groups on any of the analyzed days (Fig. 1). Even though the estimated least-squares means (LSM) may suggest an increase in $T^{*}$ over all treatments, no intragroup differences were observed. It should be pointed out that Fig. 1 shows the LSM estimates for the transformed variable analyzed with the selected statistical model, and not the actual time (expressed in sec) recorded for every group.

\section{Histopathology}

Control group sections showed no tissue alterations. Spinal cord sections of sham and lidocaine-injected animals (L05, L1 and L2) showed some polymorphonuclear cell infiltration and minor hemorrhagic areas at the injection site. This pattern corresponds to the penetration point and the path of the injection needle as was described elsewhere

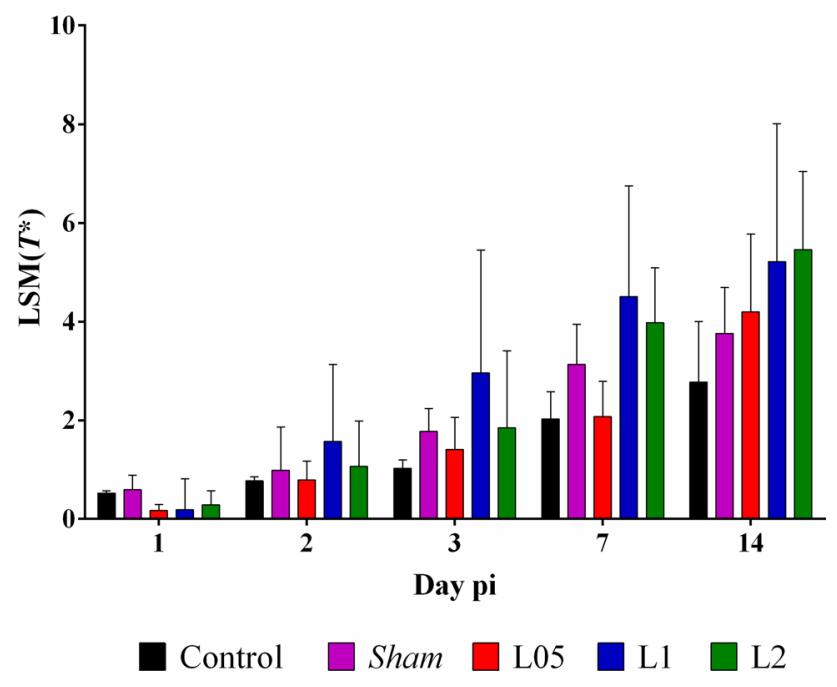

Fig. 1 Heat sensitivity test. Least-squares means (LSM) of treatments effects for $T^{*}$ at different days, as estimated by the model, expressed as LSM $\left(\mathrm{T}^{*}\right) \pm \mathrm{SEM}$
[19]. Slides of sham group showed a few swollen neurons in sections relatively closed to the entry point of the needle (from Lamina IV up to Lamina VI). Neurons of lidocaine-injected groups (L05, L1, L2) showed similar aspect to those observed in the sham group at the ipsilateral side (Fig. 2a). Nevertheless, more swollen neurons were observed as the concentration of lidocaine increased. Thus, in L2 and L1 groups, lidocaine caused more extensive inflammatory changes than in L05 group where satellitosis of glial cells was also observed. No pathological alterations at the tissue or in nervous cells were observed at the contralateral side.

Comparison of the cross-sectional areas of both sides of the C5 sections showed a significant increase of the ipsilateral side area of L2 animals in comparison to control group at pi days 1 and $2(P=0.0458$ and $P=0.0020$, respectively) (Fig. 2b, c). No significant differences were observed for the contralateral section area among groups along the experiment.

GFAP immunostained area showed a significant increase in sham animals in comparison to lidocaine injected groups and control group at pi day $1(P=0.0083$ ipsilateral; $P=0.0066$ contralateral) (Fig. $2 \mathrm{~d}$, e). At pi day 3 , sham animals showed a significant increase in the immunostained area in comparison to lidocaine injected groups $(P=0.0025$ ipsilateral; $P=0.0025$ contralateral). At pi day 7 , there was a reduction in the immunostained area of sham animals, but it was yet significantly higher when compared to L2 and L1 animals $(P=0.0010$ ipsilateral; $P=0.0021$ contralateral). Control animals showed significant differences in comparison to $\mathrm{L} 1$ animals at pi day $7(P=0.0010$ ipsilateral; $P=0.0021$ contralateral $)$ and at pi day $14(P=0.0103$ ipsilateral; $P=0.0288$ contralateral).

\section{Neuronal Counting}

Total number of neurons per section in L2 group was significantly lower than that of the rest of the groups for the ipsilateral side at pi day $3(P=0.0279)$, and significantly lower than that of the control group at pi day $7(P=0.0002)$ (Fig. 3a). L2 group was significantly different from control and sham group at pi day $14(P=0.0326)$. No significant differences were observed at the contralateral side among groups (Fig. 3b).

Estimation of the total number of neurons for the entire C5 segment was performed. No significant differences were recorded among groups except for L2, that was significantly different from the control group $(248,200 \pm 31,230)$ at pi day $7(\mathrm{~L} 2=182,250 \pm 18,450 ; P=0.0309)$ and from control and sham groups $(263,900 \pm 21,099)$ at pi day 14 $(\mathrm{L} 2=196,700 \pm 5200 ; P=0.0104)$. 

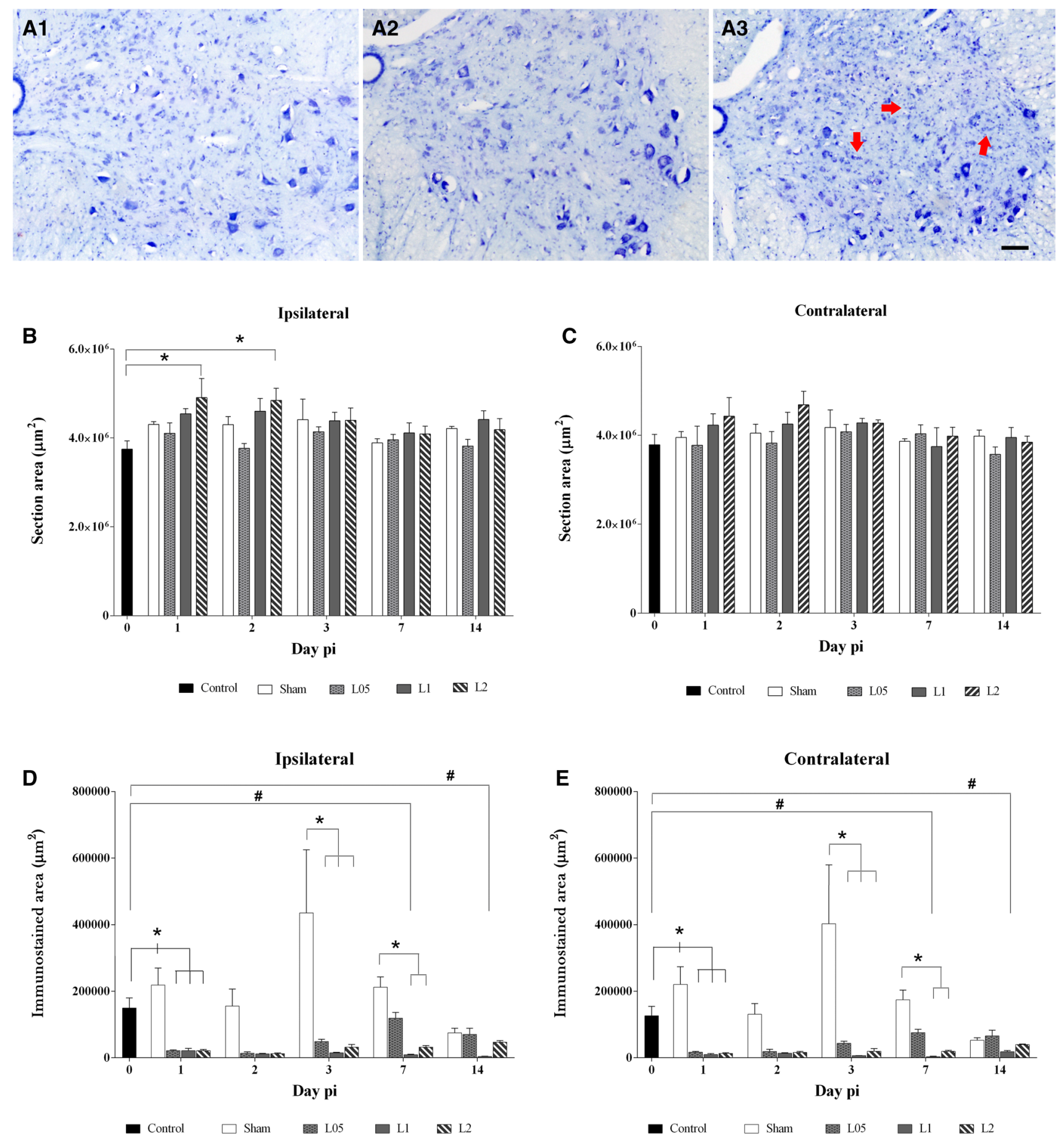

Fig. 2 Inflammatory reactivity of the spinal cord segment. a Histological aspect of a C5 spinal cord section of L05 (a1), L1 (a2) and L2 (a3) showing distribution and aspect of neurons at pi day 3. A retraction of several neurons can be observed when $1 \%(\mathbf{a} 2)$ and $2 \%(\mathbf{a 3})$ lidocaine was injected. A reduction in the number of neurons and an increase in glial reactivity can be observed (a3). Glial satellitosis can be seen surrounding degenerated neurons (arrows). Bar $100 \mu \mathrm{m}$. b Area measurement of the ipsilateral section. *L2 vs. control.

$* P<0.05$. c Area measurement of the contralateral section. No significant differences were observed. d GFAP-immunostained area at the ipsilateral side. Statistical significance: *sham against other groups. ${ }^{\#}$ Control vs. L1. ${ }^{*} \# P<0.05$. e GFAP-immunostained area at the contralateral side. Statistical significance: *of sham against the other groups. "Control vs. L1. ${ }^{*, \#} P<0.05$. In all cases, data are expressed as mean $\pm \mathrm{SEM}$ 
Fig. 3 Neuronal counting.

The number of neurons was counted based on NeuN positive staining per spinal cord section side. Data are expressed as mean \pm SEM. a Ipsilateral counting. Statistical significance: *L2 vs. control; ${ }^{*}$ L2 vs. sham $;{ }^{\&} \mathrm{~L} 2$ vs. L05 and L1.

${ }^{*, \#, \&} P<0.05$. b Contralateral counting. c Histological aspect of control C5 section. Histological aspect of L05 at $24 \mathrm{~h} \mathrm{(d)}$; $48 \mathrm{~h} \mathrm{(e);} 72 \mathrm{~h}$ (f); 7 days (g) and 14 days (h). Cresyl violet staining. Bar $=500 \mu \mathrm{m}$. Arrows point to the track of the needle used to discharge the solution
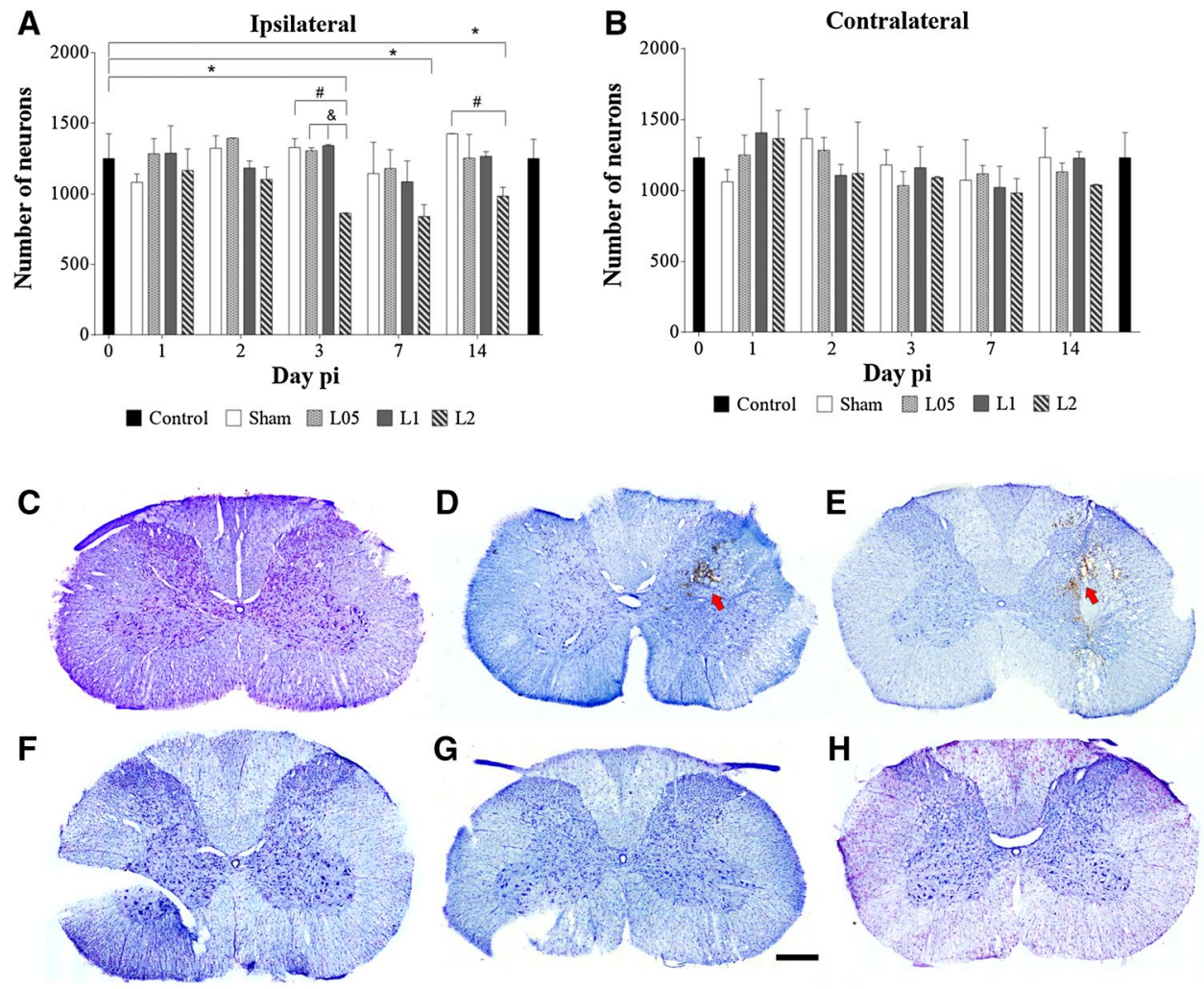

\section{Discussion}

The aim of the present work was to evaluate the structural and behavioral effects of different concentrations of lidocaine after their intraparenchymal injection into the rat cervical spinal cord. Although no significant differences between groups were found for the heat sensitivity test in the whole experiment, at pi day 1 lidocaine-treated groups showed a decrease of their latency time as compared to controls. The release of neuropeptides from sensory neurons induced by local anesthetics is thought to contribute to central sensitization in the spinal dorsal horn [20]. According to Bosshard et al. [2], concentrations of $2 \%$ lidocaine and below could be responsible for a hyperalgesic state in the experimental animals, and responses to hot plate temperature were concentration dependent being sharper for intermediate concentrations of lidocaine. Similarly, a great variability in the response of animals receiving $1 \%$ lidocaine was observed in our work. Although the latency time for all groups increased in the following days, this increase was not significant. The plantar pad of the rat has $\mathrm{A} \delta$ and $\mathrm{C}$ fibers, the former being fast conducting structures mediating fast onset of pain sensations and the latter, the slower conducting $\mathrm{C}$ fibers, mediating the long latency pain sensations. In turn, A fibers nociceptors have been classified into two types according to their responsiveness to heat stimuli [21]: type I afferent fibers, which have high activation thresholds and type II afferent fibers having lower heat threshold. Type II fibers are absent in glabrous skin. When the thermal stimulus exceeds $53{ }^{\circ} \mathrm{C}$ the average time of activation and response of type I fibers is $5 \mathrm{~s}$ [22]. On the other side, heat threshold for activating $\mathrm{C}$ fibers afferents are below pain thresholds (about $45^{\circ} \mathrm{C}$ ), suggesting that heat pain threshold depend on temporal summation of afferent activity. All these facts, added to a possible adaptation of the animals to the temperature [23] could explain the increase of the latency time observed throughout the experiment for all the groups.

Injection of $2 \%$ lidocaine significantly reduced the neuronal counting at the ipsilateral side of the $\mathrm{C} 5$ segment. The other tested concentrations of lidocaine as well as the injection of saline did not affect the neuronal counting. Several in vitro and in vivo studies have shown different dose/concentration effects of lidocaine on different cell populations, such as on cell proliferation and survival, as well as on apoptosis [24-27]. In this way, lidocaine effects on proliferation have been described as inversely proportional to the applied concentration [28]. In turn, concentration is directly proportional to the apoptotic effects [29].

Although several anti-inflammatory concentrationdependent effects of lidocaine have been described both in vitro [30], and in vivo [28, 31] in the current study we observed that injection of $2 \%$, and to a lesser extent $1 \%$ lidocaine, evoked an extent inflammatory reaction that was not observed at $0.5 \%$. We suggest that the presence of a serous 
inflammatory exudate may be responsible for the increased area observed at the ipsilateral side of affected animals. It is known that 2-20 mM lidocaine inhibits the release of leukotriene B4 in vitro, an effect that would explain the benefits of local anesthetics to prevent the formation of serous exudate [30]. Here, the $0.5 \%$ lidocaine concentration $(17 \mathrm{mM})$ would justify the absence of such an exudate. Moreover, increase of the size in the ipsilateral area of L2 rats reached its maximum at pi days 1 and 2, which might justify the subsequent decrease in the neuronal counting registered on pi day 3 .

Increase in GFAP expression is considered one of the main indicators of astrocyte reactivity [32]. In our study, the higher astrocyte reactivity was recorded in the sham group with a peak expression at pi day 3 , which is consistent with the observation of $\mathrm{Li}$ et al. [33] who described this same pattern in astrocytes 3 days after a laminectomy at the T9-T11 level. Moreover, reactivity at the contralateral side might be explained either by the release of blood or brain factors as a result of injury or by the migration of astrocytes, which would imply a previous activation [34]. Astrocytes have multiple receptors and their activity is influenced by the action of numerous cytokines. Lidocaine can reduce or inhibit the production of cytokines and interleukins involved in the activation of inflammatory processes [35, 36]. This may explain, at least in part, the reduction in the reactivity of the astrocyte population at the $\mathrm{C} 5$ segment when different concentrations of lidocaine were applied.

Differences observed between the GFAP expression of lidocaine injected groups and controls (control and sham) were also observed by Chen et al. [37]. They suggested that the mechanical injury of the nervous tissue might enhance glutamate release, which subsequently would induce astrocyte activation. Lidocaine, in turn, would reduce glutamate release and thus inhibit the activation of astrocytes [37]. Nevertheless, in the present work we only report changes in GFAP expression; more studies focusing on other glial cells are needed to determine which inflammatory mediators are involved.

To our knowledge no other studies were carried out using an intraparenchymal injection of lidocaine into the spinal cord. Nevertheless, studies using higher concentrations of lidocaine than those used in the present study have shown that the drug is highly toxic for the nervous tissue [38], and for neuronal cultures $[39,40]$. In addition, it is well known that $2.5 \%$ to $10 \%$ lidocaine concentrations induce severe cytotoxic effects even when administered by the intravenous, epidural or intrathecal route [41-43]. In vivo studies conducted by Hampl et al. [44] showed that $2 \%$ lidocaine administered by intrathecal route did not cause histopathologic or structural changes in the spinal cord, while studies performed by Ready et al. [45] reported that spinal cord histopathologic changes and neurologic deficits occurred with lidocaine concentrations beyond $8 \%$. It must be pointed out that in those reports, the percentage of lidocaine intrathecally administrated did not consider its final concentration at the targeted area. Nevertheless, the ratio between both concentrations and their effect on tissue would resemble that produced by the $0.5 \%$ and $2 \%$ lidocaine described in this report.

The intraparenchymal route allows the injection of drugs or therapeutic agents directly into the CNS and it is a promising method for the treatment of localized traumatic lesions, neurodegenerative diseases or tumors. In this work we have shown that the widely used local anesthetic lidocaine, used at $0.5 \%$ and injected by intraparenchymal via did not affect the neuronal populations of the rat spinal cord or showed pro-inflammatory effects. Therefore, we suggest a concentration of $0.5 \%$ lidocaine as an alternative treatment for localized spinal cord injuries.

Funding This work was supported by the National Agency for Promotion of Science and Technology (ANPCyT) (Grants PICT 2012-0574 to ELP and PICT 2015-2087 to FN). The authors would like to express their sincere gratitude to SAS Institute Inc for providing SAS v.9.3.1 for analyzing the data through an agreement with the College of Agriculture, University of Buenos Aires, Argentina. The expert handling of rats by Mr. H. Enrique is gratefully acknowledged.

\section{Compliance with Ethical Standards}

Conflict of interest The authors declare that they have no conflict of interest.

\section{References}

1. Turtle JD, Strain MM, Aceves M, Huang Y-J, Reynolds JA, Hook MA, Grau JW (2017) Pain input impairs recovery after spinal cord injury: treatment with lidocaine. J Neurotrauma 34(6):1200-1208. https://doi.org/10.1089/neu.2016.4778

2. Bosshard SC, Grandjean J, Schroeter A, Baltes C, Zeilhofer HU, Rudin M (2012) Hyperalgesia by low doses of the local anesthetic lidocaine involves cannabinoid signaling: an fMRI study in mice. Pain 153(7):1450-1458. https://doi.org/10.1016/j. pain.2012.04.001

3. Estebe J-P (2017) Intravenous lidocaine. Best Pract Res Clin Anaesthesiol 31(4):513-521. https://doi.org/10.1016/j. bpa.2017.05.005

4. Kobrine AI, Evans DE, LeGrys DC, Yaffe LJ, Bradley ME (1984) Effect of intravenous lidocaine on experimental spinal cord injury. J Neurosurg 60:595-601. https://doi.org/10.3171/ jns.1984.60.3.0595

5. Cole DJ, Shapiro HM, Drummond JC, Zivin JA (1989) Halothane, fentanyl/nitrous oxide, and spinal lidocaine protect against spinal cord injury in the rat. Anesthesiology 70:967-972

6. Olschewski A, Schnoebel-Ehehalt R, Li Y, Tang B, Bräu ME, Wolff M (2009) Mexiletine and lidocaine suppress the excitability of dorsal horn neurons. Anesth Analg 109:258-264. https://doi. org/10.1213/ane.0b013e3181a3d5d8

7. Tobe M, Obata H, Suto T, Yokoo H, Nakazato Y, Tabata Y, Saito S (2010) Long-term effect of sciatic nerve block with slow-release lidocaine in a rat model of postoperative pain. Anesthesiology 112:1473-1481. https://doi.org/10.1097/ALN.0b013e3181d4f66f 
8. Cheng K-I, Lai C-S, Wang F-Y, Wang H-C, Chang L-L, Ho S-T, Tsai H-P, Kwan A-L (2011) Intrathecal lidocaine pretreatment attenuates immediate neuropathic pain by modulating $\mathrm{Nav}_{1.3}$ expression and decreasing spinal microglial activation. BMC Neurol 11:71. https://doi.org/10.1186/1471-2377-11-71

9. Ineichen BV, Schnell L, Gullo M, Kaiser J, Schneider MP, Mosberger AC, Good N, Linnebank M, Schaw MB (2016) Direct, long-term intrathecal applications of therapeutics to the rodents CNS. Nat Protoc 12:104-131. https://doi.org/10.1038/nprot .2016 .151

10. Vazquez LC, Hagel E, Willenberg BJ, Dai W, Casanova F, Batich CD, Sarntinoranont M (2012) Polymer-coated cannulas for the reduction of backflow during intraparenchymal infusions. J Mater Sci Mater Med 23(8):2037-2046. https://doi.org/10.1007/s1085 6-012-4652-0

11. Lepore AC, Haenggeli C, Gasmi M, Bishop KM, Bartus RT, Maragakis NJ, Rothstein JD (2007) Intraparenchymal spinal cord delivery of adeno-associated virus IGF-1 is protective in the SOD1 ${ }^{\mathrm{G} 93 \mathrm{~A}}$ model of ALS. Brain Res 1185:256-265. https://doi. org/10.1016/j.brainres.2007.09.034

12. Rogawski MA (2009) Convection-enhanced delivery in the treatment of epilepsy. Neurotherapeutics 6(2):344-351. https://doi. org/10.1016/j.nurt.2009.01.017

13. Federici T, Hurtig CV, Burks KL, Riley JP, Krishna V, Miller BA, Sribnick EA, Miller JH, Grin N, Lamanna JJ, Boulis NM (2012) Surgical technique for spinal cord delivery of therapies: demonstration of procedure in gottingen minipigs. J Vis Exp 70:e4371. https://doi.org/10.3791/4371

14. Nishida F, Zanuzzi CN, Marquez M, Barbeito CG, Portiansky EL (2014) Vertebral trepanation as an alternative method for intraparenchymal delivery of suspensions into the spinal cord. Analecta Vet 34:11-17

15. Carter RB (1991) Differentiating analgesic and non-analgesic drug activities on rat hot plate: effect of behavioral endpoint. Pain 47:211-220

16. Portiansky EL (2013) Análisis multidimensional de imágenes digitales [online]. http://sedici.unlp.edu.ar/handle/10915/48218. Accessed 23 Aug 2018

17. Portiansky EL, Barbeito CG, Goya RG, Gimeno EJ, Zuccolilli GO (2004) Morphometry of cervical segments grey matter in the male rat spinal cord. J Neurosci Methods 139:217-229. https:// doi.org/10.1016/j.jneumeth.2004.04.031

18. Littell RC, Henry PR, Ammerman CB (1998) Statistical analysis of repeated measures data using SAS procedures. J Anim Sci 76:1216-1231

19. Nishida F, Zanuzzi CN, Martínez A, Barbeito CG, Portiansky EL (2015) Functional and histopathological changes induced by intraparenchymal injection of kainic acid in the rat cervical spinal cord. Neurotoxicology 49:68-78. https://doi.org/10.1016/j.neuro .2015.05.006

20. Leffler A, Fischer MJ, Rehner D, Kienel S, Kistner K, Sauer SK, Gavva NR, Reeh PW, Nau C (2008) The vanilloid receptor TRPV1 is activated and sensitized by local anesthetics in rodent sensory neurons. J Clin Invest 118(2):763-776. https:// doi.org/10.1172/JCI32751

21. Treede R-D, Meyer RA, Raja SN, Campbell JN (1995) Evidence for two different heat transduction mechanisms in nociceptive primary afferents innervating monkey skin. J Physiol 483.3:747-758

22. Schepers RJ, Ringhamp M (2010) Thermoreceptors and thermosensitive afferents. Neurosci Biobehav Rev 177-184. https://doi. org/10.1016/j.neubiorev.2009.10.003

23. Luukko M, Konttinen Y, Kemppinen P, Pertovaara A (1994) Influence of various experimental parameters on the incidence of thermal and mechanical hyperalgesia induced by a constriction mononeuropathy of the sciatic nerve in lightly anesthetized rats. Exp Neurol 128(1):143-154. https://doi.org/10.1006/exnr.1994.1122
24. Jejurikar SS, Welling TH, Zelenock JA, Gordon D, Burkel WE, Carlson BM, Messina LM (1997) Induction of angiogenesis by lidocaine and basic fibroblast growth factor: a model for in vivo retroviral-mediated gene therapy. J Surg Res 67:137-146. https ://doi.org/10.1006/jsre.1996.4989

25. Lei B, Cottrell JE, Kass IS (2001) Neuroprotective effect of low-dose lidocaine in a rat model of transient focal cerebral ischemia. Anesthesiology 95:445-451

26. Lenfant F, Lahet J-J, Chaillot B, Freysz M (2004) The protective effects of lidocaine on human erythrocytes stored for seven days at 04 degrees C. Cell Mol Biol Lett 9:301-304

27. Quinteros Villarruel E, Orman B, Borda E (2014) Lidocaineinduced cell growth of human gingival fibroblasts. Role of $\mathrm{Na}^{+}-\mathrm{K}^{+}$-ATPase and PKC activities. Pharmacol Pharmacy 5:796-805. https://doi.org/10.4236/pp.2014.58090

28. Portiansky EL, González PH (1995) Protective effect of lidocaine in the experimental foot-and-mouth disease pancreatitis. Experientia 51:1060-1062

29. Quinteros Villaruel E, Borda E, Sterin-Borda L, Orman B (2011) Lidocaine-induced apoptosis of gingival fibroblasts: participation of cAMP and PKC activity. Cell Biol Int 35:783-788. https://doi.org/10.1042/CBI20100200

30. Hollmann MW, Durieux ME (2000) Local anesthetics and the inflammatory response: a new therapeutic indication? Anesthesiology 93:858-875

31. Gallos G, Jones DR, Nasr SH, Emala CW, Lee HT (2004) Local anesthetics reduce mortality and protect against renal and hepatic dysfunction in murine septic peritonitis. Anesthesiology 101:902-911

32. Haim LB, Carrillo-de Sauvage MA, Ceyzériat K, Escartin C (2015) Elusive roles for reactive astrocytes in neurodegenerative diseases. Front Cell Neurosci 9:278. https://doi.org/10.3389/ fncel.2015.00278

33. Li G, Cao Y, Shen F, Wang Y, Bai L, Guo W, Bi Y, Lv G, Fan $\mathrm{Z}$ (2016) Mdivi-1 inhibits astrocyte activation and astroglial scar formation and enhances axonal regeneration after spinal cord injury in rats. Front Cell Neurosci 10:241. https://doi. org/10.3389/fncel.2016.00241

34. Jegliński W, Koczyk D, Zaremba M, Oderfeld-Nowak B (1995) Bilateral gliosis in unilaterally lesioned septohippocampal system: changes in GFAP immunoreactivity and content. J Neurosci Res 15:41:394-402. https://doi.org/10.1002/jnr.490410312

35. Su D, Gu Y, Wang Z, Wang X (2010) Lidocaine attenuates proinflammatory cytokine production induced by extracellular adenosine triphosphate in cultured rat microglia. Anesth Analg 111:768-774. https://doi.org/10.1213/ANE.0b013e3181e9e897

36. Chiu KM, Lu CW, Lee MY, Wang MJ, Lin TY, Wang SJ (2016) Neuroprotective and anti-inflammatory effects of lidocaine in kainic acid-injected rats. Neuroreport 27:501-507. https://doi. org/10.1097/WNR.0000000000000570

37. Chen JJ, Lue JH, Lin LH, Huang CT, Chiang RP, Chen CL, Tsai YJ (2010) Effects of pre-emptive drug treatment on astrocyte activation in the cuneate nucleus following rat median nerve injury. Pain 148:158-166. https://doi.org/10.1016/j. pain.2009.11.004

38. Kim DD, Asif A, Kataria S (2016) Presentation of neurolytic effect of $10 \%$ lidocaine after perineural ultrasound guided injection of a canine sciatic nerve: a pilot study. Korean J Pain 29:158 163. https://doi.org/10.3344/kjp.2016.29.3.158

39. Johnson ME, Saenz JA, DaSilva AD, Uhl CB, Gores JG (2002) Effect of local anesthetic on neuronal cytoplasmic calcium and plasma membrane lysis (necrosis) in a cell culture model. Anesthesiology 97:1466-1476

40. Zheng X, Chen F, Zheng T, Huang F, Chen J, Tu W (2016) Amitriptyline activates TrkA to aid neuronal growth and attenuate anesthesia-induced neurodegeneration in rat dorsal root 
ganglion neurons. Medicine (Baltimore) 95(18):e3559. https:// doi.org/10.1097/MD.0000000000003559

41. Cherng C-H, Wong C-S, Wu C-T, Yeh C-C (2011) Glutamate release and neurologic impairment after intrathecal administration of lidocaine and bupivacaine in the rat. Reg Anesth Pain Med 36:452-456. https://doi.org/10.1097/AAP.0b013e318228cdb0

42. Muguruma T, Sakura S, Saito Y (2006) Epidural lidocaine induces dose-dependent neurologic injury in rats. Anesth Analg 103:876881. https://doi.org/10.1213/01.ane.0000237287.53957.18

43. Zhao G, Ding X, Guo Y, Chen W (2014) Intrathecal lidocaine neurotoxicity: combination with bupivacaine and ropivacaine and effect of nerve growth factor. Life Sci 112:10-21. https://doi. org/10.1016/j.lfs.2014.07.003

44. Hampl K, Steinfeldt T, Wulf H (2014) Spinal anesthesia revisited: toxicity of new and old drugs and compounds. Curr Opin Anaesthesiol 27:549-555. https://doi.org/10.1097/ACO.0000000000 000108

45. Ready LB, Plumer MH, Haschke RH, Austin E, Sumi SM (1985) Neurotoxicity of intrathecal local anesthetics in rabbits. Anesthesiology 63:364-370 